\title{
Fracture Resistance of Yttria-Stabilized Zirconia Dental Implant Abutments
}

\author{
Nimet D. Adatia, DDS, MS, ${ }^{1}$ Stephen C. Bayne, MS, PhD, ${ }^{2}$ Lyndon F. Cooper, DDS, MS, PhD, ${ }^{1}$ \\ \& Jeffery $\mathrm{Y}$. Thompson, $\mathrm{PhD}^{3}$ \\ 1 University of North Carolina School of Dentistry, Chapel Hill, NC \\ 2 University of Michigan School of Dentistry, Ann Arbor, MI \\ ${ }^{3}$ Dental School, University of Texas Health Science Center at San Antonio, TX
}

\author{
Keywords \\ Implant abutments; static loading; angled \\ loading; zirconia; abutment complication. \\ Correspondence \\ Nimet D. Adatia, 19 Coral Reef Link NE, \\ Calgary, AB, T3J 3Y5, Canada. E-mail: \\ adatian@gmail.com \\ Supported in part by an ACP/3M ESPE \\ Fellowship in Geriatric Prosthodontics \\ (Awarded 2004). Presented as a poster \\ presentation at the IADR 84th General \\ Session, Brisbane, Australia, June 28 \\ to July 1, 2006. Read at ACP Annual Session, \\ Miami, FL, November 2006.
}

Accepted November 8, 2007

doi: 10.1111/j.1532-849X.2008.00378.x

\begin{abstract}
Purpose: An in vitro study was performed to assess the effect of different degrees of clinical reduction of zirconia abutments on the failure load of clinical assemblies. Materials and Methods: Zirconia abutments (Y-TZP Ceramic Abutment, Astra Tech) were prepared with $0,0.5$, or $1 \mathrm{~mm}$ of external axial reduction starting $1 \mathrm{~mm}$ above the height-of-contour. Abutments $(\mathrm{n}=10)$ were attached to implant analogs $(25 \mathrm{Ncm}$ torque) embedded in a stainless steel cylinder using Field's metal. Fracture loads (N) were determined when assemblies were loaded at $60^{\circ}$ off-axis until failure (Instron, CHS $=0.1 \mathrm{~mm} / \mathrm{min})$. Groups were statistically compared using ANOVA $(p<0.05)$. Results: Fracture loads for all assemblies displayed large variations within groups. There were no statistically significant differences $(p>0.05)$ among different abutment groups with a mean fracture load of $429 \mathrm{~N}( \pm 140)$ for the control group, $576 \mathrm{~N}( \pm 120)$ for $0.5-\mathrm{mm}$ margins, and $547( \pm 139)$ for 1.0-mm margins. All fractures occurred at the interface where the abutment was connected to the analog.

Conclusion: In this in vitro study of simulated ultimate assembly strength, the preparation of zirconia abutments did not significantly impair the fracture resistance of simulated implant assemblies. All implant abutments fractured at rates higher than the maximum incisal forces (90-370 N) estimated to occur in the anterior region of the mouth.
\end{abstract}

Contemporary expectations for dental implant performance include the delivery of esthetically pleasing restorations. Among the factors affecting dental implants esthetics is the choice of abutment type and material. Often, prefabricated components cannot provide refined morphologic enhancement of dental implant esthetics, and modification by additive or subtractive methods is required. One option for esthetic dental implant restorations is the selection and modification of a zirconia abutment.

While other materials including titanium, cast gold, and alumina can be used to create abutments of custom form through preparation, ${ }^{1}$ titanium abutments can create an unesthetic blue hue from light reflections of titanium shining through the tissues. ${ }^{1-3}$ Abutments of milled or as-sintered alumina overcome this esthetic problem ${ }^{4,5}$ and provide favorable biocompatibility, perhaps illustrated by fibroblast adherence. ${ }^{6}$ Alumina and now zirconia abutments are of increasing popularity because of their light transmittance quality and color, as well as the reported high fracture resistance ${ }^{7-11}$ however, alumina fracture resistance $(239 \pm 83 \mathrm{~N})$ is poor compared to titanium $(324 \pm$
$85 \mathrm{~N})$ or zirconia $(294 \pm 53 \mathrm{~N}) .^{12-19}$ The reported strength of zirconia abutments reflects the manufactured component strength and requires examination of the clinical strength represented by the modified zirconia abutment. While an absolute minimal strength for abutments has not been defined, it is assumed that abutments should offer resistance to functional loading. Peak values for occlusal force in the incisal area are reported in the range of 90 to $370 \mathrm{~N} .^{13,14} \mathrm{~A}$ durable, esthetic restoration should resist such forces.

Although zirconia abutments for intraoral preparation are available for clinical use, there have been very few laboratory studies ${ }^{1,12,15}$ investigating the fracture resistance of zirconia abutment assemblies. When unmodified zirconia abutments were tested to failure, they failed in the cervical portion of the abutment, adjacent to the gold screw and implant platform. ${ }^{1,15}$ The implant-abutment interface design plays a role in the observed failure. Internal implant abutment connections more widely distribute forces along the interface as compared with external hex designs. ${ }^{16,17}$ The aim of this investigation was to determine the effect of clinical abutment reduction 
on the strength of assemblies containing yttrium-stabilized zirconia abutments (Astra Tech, Inc., Waltham, MA).

\section{Methods and materials}

Thirty zirconia abutments (4.5/5.0 diameter, $5.5 \mathrm{~mm}$, Astra Tech) were randomly divided into three groups of ten abutments:

(1) Group 1 was tested without modification (i.e., control).

(2) Group 2 was modified to include a chamfer margin of $0.5 \mathrm{~mm}, 1 \mathrm{~mm}$ above the abutment height of contour, and $2.0 \mathrm{~mm}$ of occlusal reduction.

(3) Group 3 was modified to include a chamfer margin of $1.0 \mathrm{~mm}, 1 \mathrm{~mm}$ above the abutment height of contour, and $2.0 \mathrm{~mm}$ of occlusal reduction.

All preparations were made using coarse diamonds (Twostriper, Premier, Plymouth Meeting, PA, 515.7, ISO 110, Lot 330) in a high-speed dental handpiece, under conditions of continuous air/water spray. All abutments were prepared by a single operator. Following preparation, the abutments were assembled with the analogs in stainless steel test mounts using titanium abutment screws that accompany each abutment, torqued to $25 \mathrm{Ncm}$ using a dental implant torque wrench. Following modification, the dimension of each abutment was measured with a Boley gauge at the incisal, middle, and gingival third and was recorded. Abutments $(\mathrm{n}=30$, lots 21289, $22902)$ and analogs $(n=5$, lot 12207$)$ were provided by the Astra Tech.

For testing, each implant analog was first positioned with a stainless steel cylinder with the analog long axis parallel to the cylinders' long axes and fixed in place using Field's metal alloy (www.scitoys.com). Abutments were then connected to implant analogs for testing as described above. The implant analog/abutment screw/abutment combination is hereafter referred to as the assembly (Fig 1). The cylinders with assemblies were placed onto a stainless steel holding apparatus, which was inclined at $30^{\circ}$ to the vertical, enabling loading at $60^{\circ}$ off-axis in a universal testing machine (Instron, Model 4411, Grove City, PA). To prevent inadvertent surface damage by the loading stylus on the zirconia abutment and to further control loading, a thin layer $(0.1 \mathrm{~mm})$ of Mylar film was inserted between the stylus and the abutment. A vertical load was applied to the incisal edge (crosshead speed $=0.1 \mathrm{~mm} / \mathrm{min}, 25^{\circ} \mathrm{C}$ ) until failure. Fracture of the abutment was accompanied by an audible pop. Specimens were tested in order of groups, without special order within the groups.

The mean fracture loads for each group of prepared assemblies were compared ( $\alpha \leq 0.05$, one-way ANOVA) using personal computer software (Analyse-It, www.analyse-it.com, Leeds, UK). A regression analysis was used to determine the amount of abutment reduction that could be achieved while maintaining acceptable fracture resistance.

Correlation among the calculated volumes of remaining material and fracture strength was assessed. Abutment volumes from the preparation line to the incisal portion were determined using the formula for a truncated cone, $V=(\pi \mathrm{h} / 12)\left(\mathrm{D}^{2}+\mathrm{Dd}+\right.$ $d^{2}$ ), where $V$ is the truncated cone volume, $h$ is the height of

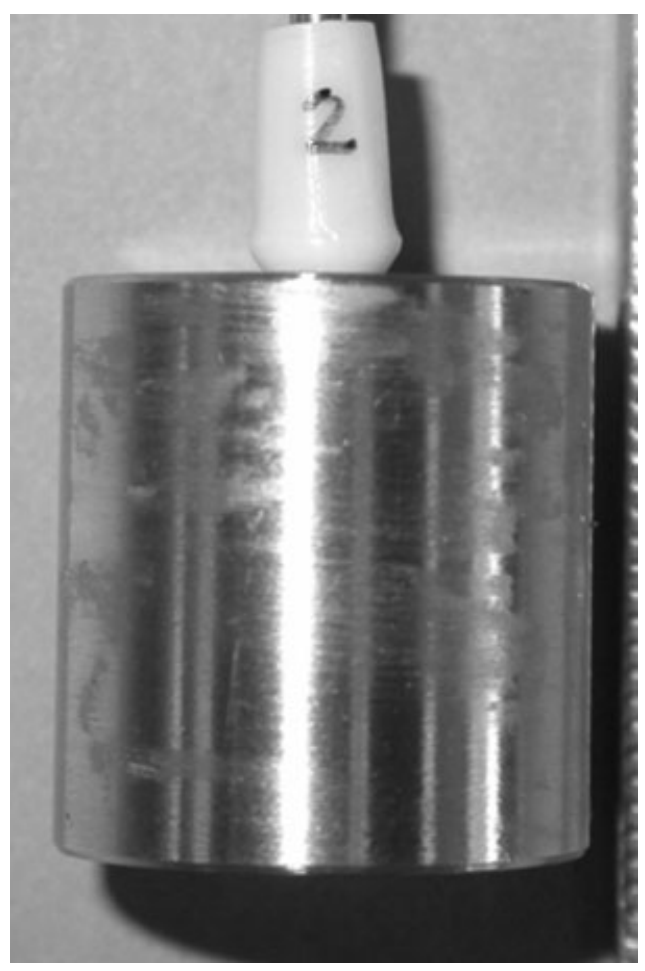

Figure 1 Abutment assembly fixed within implant analog with Field's metal. The analog was fixed flush with the top of the stainless steel cylinder and held in place with Field's metal. The prepared abutments were then screwed in place with the screw provided and torqued to $25 \mathrm{Ncm}$. This is now considered the "assembly."

the truncated cone, $\mathrm{D}$ is the diameter of the base, and $\mathrm{d}$ is the diameter of the top of the cone. The abutment was assumed to be two stacked, truncated cones with the top cone starting at the prepared portion of the abutment. No correction was made for the internal threaded portion or the tapering ceramic tail of the abutment.

\section{Results}

The preparation of zirconia abutments was achieved without material failure; however, during preparation of the zirconia abutments, the zirconia seemed to wear the metal bur, and sparks were also commonly observed. The actual amount of axial reduction for individual abutments was measured (Table 1), and mean volumes of reduction were calculated (Table 2). Fracture loads for all assemblies displayed large variations within groups. There were no statistically significant differences $(p>0.05)$ among different abutment groups. There was no correlation $\left(r^{2}=0.0619\right)$ among the calculated volumes of remaining material and fracture strength.

Assembly using torque controllers also occurred without damage to the unmodified or modified abutments. During the testing procedures, all screws became loose, despite being tightened to $25 \mathrm{Ncm}$ immediately prior to loading. Fracture occurred for all abutments, and all fractures occurred 
at the abutment/analog interface (Fig 2). Analogs were inspected visually after each run to ensure no deformation occurred and were no longer used when deformation was suspected.

A specific pattern of load-to-failure was demonstrated by all of the specimens tested. A typical direct loading curve for a prepared abutment assembly revealed four distinct regions. The first region, representative of initial loading of the assembly, indicates deformation of the abutment. The second region was attributed to deformation of the abutment screw. The third region represented abutment fracture. The fourth aspect of the curve represents the load returning to zero after the abutment fracture (Fig 3). Preparation of the abutment did not change this general pattern. The loads present at the inflection points representing these regions were measured and attributed to a contribution to the assembly by the abutment, screw, or the implant analog (Fig 4). Although the actual dissection of the events in the assembly tests could not be done, the distinct regions and inflection points are extrapolated by the authors. No conclusions are actually being drawn from this respect.

\section{Discussion}

The main finding of this study was that the overall strength of the assemblies including zirconia abutments was not affected by the wet chamfer preparation of the abutment. There was no statistically significant difference in the average values. The average values of each group were at least $15 \%$ higher than the highest average force reported in the literature $(370 \mathrm{~N}$ vs. $435 \mathrm{~N}) .{ }^{13,14}$ Failure of the abutments occurred in the model at forces that exceed typically cited values for anterior tooth

Table 1 Mean reduction of abutments in each specimen group as measured by Boley gauge after marginal reduction with a diamond

Mean reduction $(\mathrm{mm})$

\begin{tabular}{lccc}
\cline { 2 - 4 } Specimen aim & Cervical region & Middle region & Incisal region \\
\hline 0.5-mm margin & $0.5 \pm 0.1$ & $0.38 \pm 0.1$ & $0.62 \pm 0.2$ \\
$1.0-\mathrm{mm}$ margin & $0.8 \pm 0.1$ & $0.72 \pm 0.2$ & $0.94 \pm 0.2$ \\
\hline
\end{tabular}

Reduction was measured at the incisal, middle, and cervical thirds. Measurements were then compared to the group aim of reduction.

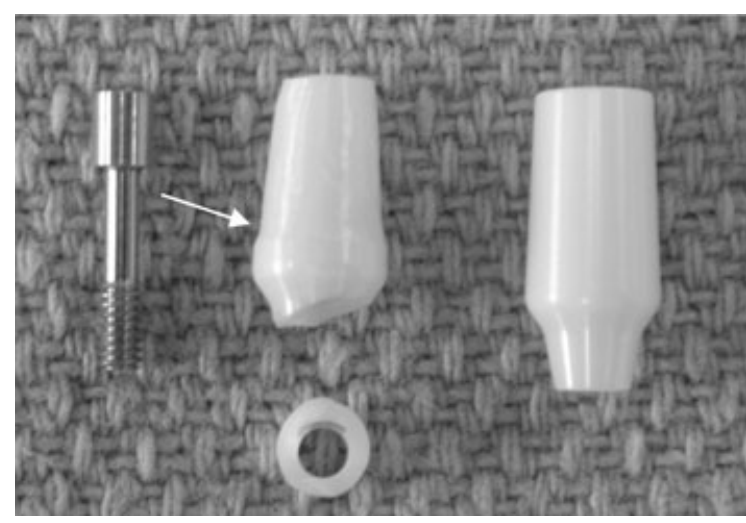

Figure 2 Typical abutment fracture. Arrow indicates where the margin design is on this $0.5-\mathrm{mm}$ specimen. The unprepped abutment and screw are shown prior to testing. Note the fracture is below the margin preparation, at the thinnest portion of the abutment.

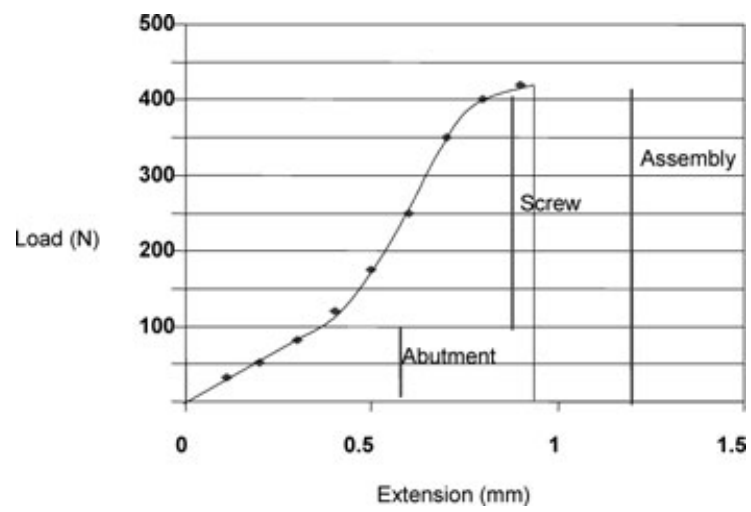

Figure 3 Schematic representation of typical load-deformation curve (vertical lines indicate the different assembly parts seemingly associated with the major deformations). There are four distinct regions. The first region, representative of initial loading of the assembly, indicates deformation of the abutment. The deflection point leads into the second region, which was attributed to deformation of the abutment screw. The next deflection point leads into the third region, representing abutment fracture. The fourth aspect of the curve represents the load returning to zero after the abutment fracture. Preparation of the abutment did not change this general pattern.

function and for relevant all-ceramic crowns. This in vitro estimate of preparation effects on the zirconia abutment strength suggests that modest preparation for chamfer design crowns is possible.

Table 2 Calculated mean volumes of the abutments and strengths for each group

\begin{tabular}{lccccc}
\hline Group & $\mathrm{n}$ & Abutment volume $\left(\mathrm{mm}^{3}\right)$ & Abutment strength (N) & Screw strength (N) & Assembly strength (N) \\
\hline Control & 10 & $150 \pm 0$ & $282 \pm 59$ & $246 \pm 111$ & $429 \pm 140$ \\
$0.5 \mathrm{~mm}$ & 10 & $125 \pm 6$ & $205 \pm 62$ & $371 \pm 123$ & $576 \pm 120$ \\
$1.0 \mathrm{~mm}$ & 10 & $108 \pm 7$ & $172 \pm 48$ & $375 \pm 110$ & $547 \pm 139$ \\
\hline
\end{tabular}

The abutment and screw strengths were extrapolated from their load deformation curves. The final assembly strengths were also taken from the load-deformation curve just as the abutment broke. 
Figure 4 Peak load for each portion of the load-deformation curve depicted in Figure 3 for each assembly group. The strengths for the abutment and screw were extrapolated from the load-deformation curves for each group. It can be seen that as the abutment is prepared, the actual strength of the abutment will decrease. The screws in the prepared groups both have the same strength values. The assembly values are not significantly different from each other $(p>0.5)$ when the system works as a whole.

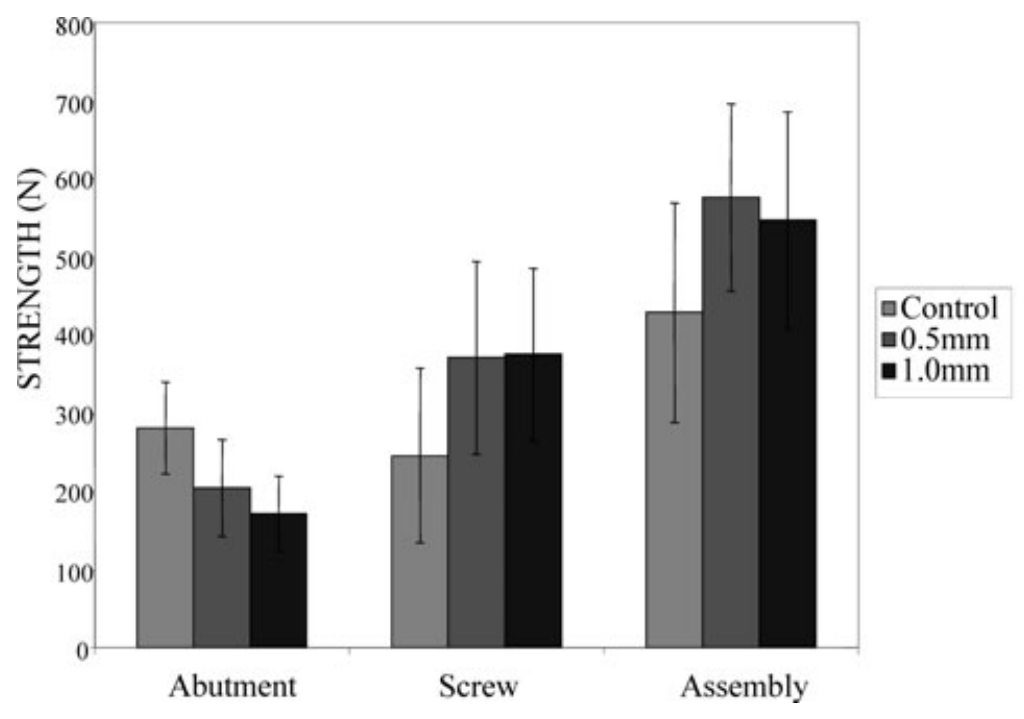

This general conclusion requires careful consideration of the present model system. First, this model tested a dental material (zirconia) in a static manner. Second, the abutment assembly was oriented to test only the fracture of the zirconia abutment. The results, however, showed that the screw inside the abutment might have played a considerable role in the final strength values. During preparation of the specimens, an effort was made to ensure that the amount of material removed was uniform and that all specimens were treated equally. Abutments provided by the manufacturer for these tests did have different lot numbers but were presumed to be identical.

Hand preparation was controlled as practically as feasible; however, variability in the data may reflect the reality of clinical abutment preparation (e.g., duration, force, and direction of preparation, diamond bur conditions, single operator). In addition, the simulation used stainless steel implant analogs instead of titanium implants. Analog failure was not observed by SEM, and internal deformation of the analog may have occurred prior to visualization. Irrespective of these concerns, screw deformation suggests the abutment screw was the first element of the assembly affected by this progressive loading protocol.

Some authors have reported that the use of gold screws and a controlled torque should reduce the rate of failure as com- pared to titanium screws. ${ }^{12,19}$ Here, a titanium retaining screw provided by the manufacturer was used throughout the current study. This was the original hexed screw with a square head (Fig 5). A previous study found that in $63 \%$ of specimens, abutment screw deformation occurred, despite the use of a gold screw. ${ }^{12}$ It was also reported that no screw loosening was found after fatiguing the zirconia specimens. Fracture loads reported for fatigued zirconia abutments were $294 \pm 53 \mathrm{~N} .{ }^{12}$ In the present study, all titanium screws were loose after static loads were applied. This could be due to the plastic deformation of the titanium screw occurring over a longer period of time (cross head speed $(\mathrm{CHS})=0.1 \mathrm{~mm} / \mathrm{min}$ compared to $1.5 \mathrm{~mm} / \mathrm{min}),{ }^{12}$ as well as other factors, such as the lack of an excellent fit into the analog.

Yildirim et $\mathrm{al}^{1}$ tested external hex implant connections, and Mitsias ${ }^{15}$ tested conical seal design implant connections, and both found that when zirconia abutment assemblies failed, they failed at the cervical portion of the abutment, near the gold screw and platform of the implant. This area has been presumed to be an area of the highest torque and stress concentrations due to the levering effects. ${ }^{1}$ A common location of failure near the screw head seat in the abutment is suggested by the current data. Chamfer preparation of the abutment
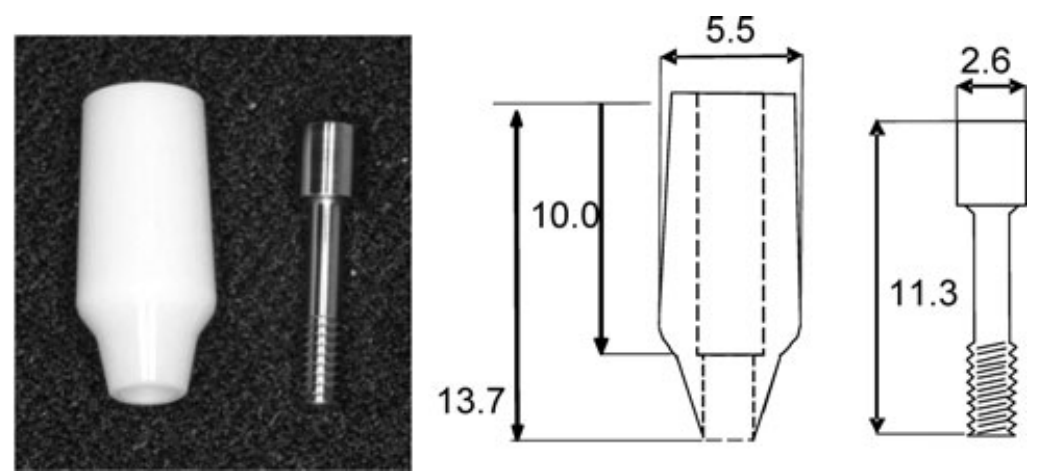
did not affect the location or force magnitude required for failure.

This investigation did not include a full veneer crown in the model system. Other authors ${ }^{1}$ included crowns over the zirconia abutments and found the zirconia abutment failed in $40 \%$ of the specimens prior to either the all-ceramic crown fracturing or the gold screw bending. A crown may act to stress shield the abutment from the effects of the load, thereby allowing a larger load to be applied before failure is noted, although the effects of the cement and restoration on stress-shielding and/or blunting of any surface defects on the abutment were not examined in this study. This could explain the lower forces resulting in failure in this study than in previous reports (from 547 to $788 \mathrm{~N}$ ). ${ }^{1,15}$

There is concern that microdamage accumulation during abrasive reduction of zirconia abutments could account for the reduced strength and be a cause of clinical failure. In this evaluation, high speed, water-cooled diamond bur preparation of the abutments was performed. It has been argued that water irrigation has the effect of increased preparation efficiency by cleaning the debris away from the abrasive particles on diamonds. ${ }^{20}$

The extrapolated flexure strength of the control group of the zirconia abutment was not statistically different from either of the experimental groups; however, its values were considerably higher. Calculations were done to correct the results on the basis of the total remaining volume of the abutment versus the flexural strength; however, no correlation was observed $\left(\mathrm{r}^{2}=\right.$ 0.0619).

There have been no published clinical trials evaluating the clinical outcomes of therapy using zirconia conical seal design abutments; however, a clinical trial using the CerAdapt alumina abutment found that $12 \%$ of the abutments failed before loading, and $7 \%$ failed after 7 months. The same clinical trial reported $100 \%$ success with CeraOne titanium abutments. ${ }^{5}$ Ultimately, concerns regarding the clinical utility of a zirconia abutment require long-term clinical evaluation. At present, the use of a zirconia abutment that includes modification may be considered feasible. Modification using wet, high-speed preparation is advocated.

Finally, it would be worthwhile to examine the fatigue behavior of this assembly at loads much lower than the failures observed here. Repetitive cycling at low loads produces small amounts of plastic deformation that accumulate over many cycles and produce failures far below the static elastic limit of a material or assembly. Fatigue cycling of the current assembly from loads $0-40 \%$ of failure levels $(\sim 200 \mathrm{~N})$ for several million cycles would be the ideal approach to examine this effect, but this experiment is not practical. Alternatively, the assembly could be loaded for 100,000 cycles at $1 \mathrm{cycle} / \mathrm{sec}$, which would only take a few days, and then tested to failure to disclose the impact on altering the original load-deformation failure curve for the assembly. This type of analysis has been used before for testing assemblies of cemented crowns. ${ }^{21}$

\section{Conclusions}

Within the limitations of these experiments, the following could be concluded:
(1) Margin preparation, with irrigation, of the tested abutments up to $1.0 \mathrm{~mm}$ did not adversely affect the fracture strength of abutment assemblies.

(2) The weakest point of the abutment assemblies seemed to be the abutment/analog interface.

\section{Acknowledgments}

The authors gratefully acknowledge Astra Tech for materials contributed, as well as Drs. David Felton and Erica Texiera for all their support.

\section{References}

1. Yildirim M, Fischer H, Marx R, et al: In vivo fracture resistance of implant-supported all-ceramic restorations. J Prosthet Dent 2000;90:325-331

2. Tan PLB, Dunne JT: An esthetic comparison of metal ceramic crown and cast metal abutment with an all-ceramic crown and zirconia abutment: a clinical report. J Prosthet Dent 2004;91:215-218

3. Brodbeck U: The ZiReal Post: a new ceramic implant abutment. J Esthet Restor Dent 2003;15:10-23

4. Vigolo P, Fonzi F, Majzoub Z, et al: An in vitro evaluation of ZiReal abutments with hexagonal connection: in original state and following abutment preparation. Int J Oral Maxillofac Implants 2005;20:108-114

5. Andersson B, Taylor A, Lang B, et al: Alumina ceramic abutments used for single-tooth replacement: a prospective 1-3 year multicenter study. Int J Prosthodont 2001;14:432-438

6. Mustafa K, Oden A, Wenneberg A, et al: The influence of surface topography of ceramic abutments on the attachement and proliferation of human oral fibroblasts. Biomaterials 2005;26:373-381

7. Guazzato M, Quach L, Albakry M, et al: Influence of surface and heat treatments on the flexural strength of Y-TZP dental ceramic. J Dent 2005;33:9-18

8. Kosmac T, Oblak C, Jevnikar P, et al: Strength and reliability of surface treated Y-TZP dental ceramics. J Biomed Mater Res 2000;53:304-313

9. Kosmac T, Oblak C, Jevnikar P, et al: The effect of surface grinding and sandblasting on flexural strength and reliability of Y-TZP zirconia ceramic. Dent Mater J 1999;15:426-433

10. Luthardt RG, Holzhuter M, Sandkuhl O, et al: Reliability and properties of ground Y-TZP-zirconia ceramics. J Dent Res 2002;81:487-491

11. Zhang Y, Lawn B, Rekow ED, et al: Effect of sandblasting on the long-term performance of dental ceramics. J Biomed Mater Res Part B: Appl Biomater 2004;71:381-386

12. Butz F, Heydecke G, Okutan M, et al: Survival rate, fracture strength and failure mode of ceramic implant abutments after chewing simulation. J Oral Rehabil 2005;32:838-843

13. Paphangkorakit J, Osborn JW: The effect of pressure on maximum incisal bite force in man. Arch Oral Biol 1997;42:11-17

14. Haraldson T, Carlsson GE, Ingervall B: Functional state, bite force and postural muscle activity in patients with osseointegrated oral implant bridges. Acta Odontol Scand 1979;37:195-206

15. Mitsias, ME: Fatigue test analysis of two esthetic implant abutments. 2004; p1-92. MS Thesis, Department of Biomaterials and Biomimetics, Graduate School of Arts and Sciences, New York University. 
16. Maeda Y, Satoh T, Sogo M: In Vitro differences of stress concentrations for internal and external hex implant-abutment connections: a short communication. J Oral Rehabil 2006;33:75-78

17. Khraisat A, Stegaroiu R, Nomura S, et al: Fatigue resistance of two implant/abutment joint designs. J Prosthet Dent 2002;88:604-610

18. Tripodakis AP, Strub JR, Kappert HF, et al: Strength and mode of failure of single implant all-ceramic abutment restorations under static load. Int J Prosthodont 1995; $8: 265-272$
19. Strub JR, Gerds T: Fracture strength and failure mode of five different single-tooth implant-abutment combinations. Int J Prosthodont 2003;16:167-171

20. Blue DS, Griggs JA, Woody RD, et al: Effects of bur abrasive particle size and abutment composition on preparation of ceramic implant abutments. J Prosthet Dent 2003;90:247254

21. Zarb GJA: Mechanical fatigue of two dental luting agents in simulated function. 1999. Masters Thesis, Department of Prosthodontics, School of Dentistry, University of North Carolina, Chapel Hill, NC 